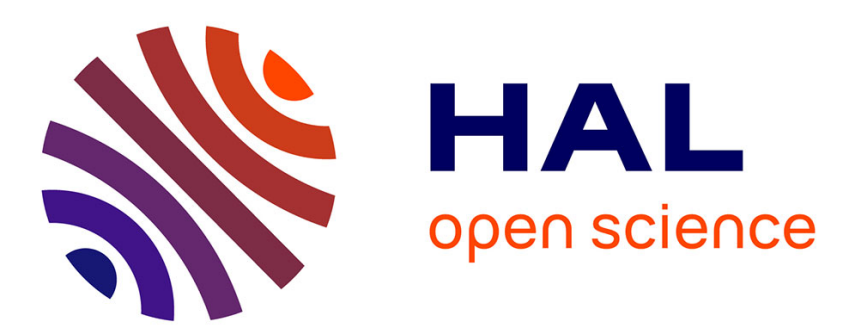

\title{
Active bundles of polar and bipolar filaments
}

F. Kreten, Ch. Hoffmann, Daniel Riveline, K. Kruse

\section{To cite this version:}

F. Kreten, Ch. Hoffmann, Daniel Riveline, K. Kruse. Active bundles of polar and bipolar filaments. Physical Review E , 2018, 98 (1), 10.1103/PhysRevE.98.012413 . hal-02398899

\section{HAL Id: hal-02398899 \\ https://hal.science/hal-02398899}

Submitted on 22 Dec 2020

HAL is a multi-disciplinary open access archive for the deposit and dissemination of scientific research documents, whether they are published or not. The documents may come from teaching and research institutions in France or abroad, or from public or private research centers.
L'archive ouverte pluridisciplinaire HAL, est destinée au dépôt et à la diffusion de documents scientifiques de niveau recherche, publiés ou non, émanant des établissements d'enseignement et de recherche français ou étrangers, des laboratoires publics ou privés. 


\title{
Active bundles of polar and bipolar filaments
}

\author{
F. H. Kreten ${ }^{*}$ and Ch. Hoffmann ${ }^{\dagger}$ \\ Theoretische Physik, Universität des Saarlandes, 66123 Saarbrücken, Germany \\ D. Riveline ${ }^{\ddagger}$ \\ Laboratory of Cell Physics ISIS/IGBMC, CNRS and University of Strasbourg, Strasbourg, France; \\ Institut de Génétique et de Biologie Moléculaire et Cellulaire, Illkirch, France; \\ Centre National de la Recherche Scientifique, UMR7104, Illkirch, France; \\ Institut National de la Santé et de la Recherche Médicale, U964, Illkirch, France; \\ and Université de Strasbourg, Illkirch, France \\ K. Kruse ${ }^{\S}$ \\ NCCR Chemical Biology, Departments of Biochemistry and Theoretical Physics, University of Geneva, 1211 Geneva, Switzerland
}

(Received 21 February 2018; published 23 July 2018)

\begin{abstract}
Bundles of actin filaments and molecular motors of the myosin family are a common subcellular organizational motif. Typically, such bundles are under contractile stress resulting from interactions between the filaments and the motors. This holds in particular for contractile rings that appear in the late stages of cell division in animal cells and that cleave the mother into two daughter cells. It was recently shown that myosin organizes into regularly spaced clusters along rings in mammalian cells, whereas myosin clusters in fission yeast travel along the perimeter of actomyosin rings [Wollrab et al., Nat. Commun. 7, 11860 (2016)]. A mechanism based on the association of the structurally polar actin filaments into bipolar structures was shown to provide a common explanation for both observations. Here, we analyze the dynamics of this mechanism in detail. We find a rich phase diagram depending on the actomyosin interaction strength and the stability of the bipolar structures. The system can notably organize into traveling waves. Furthermore, we identify the nature of the bifurcations connecting the various patterns as parameters are changed. Finally, we report experimental patterns observed in cytokinetic rings in fission yeast and link them to solutions of our dynamic equations. Our analysis highlights the possible role played by local polarity sorting of actin filaments for the dynamics and functionality of actomyosin networks.
\end{abstract}

DOI: 10.1103/PhysRevE.98.012413

\section{INTRODUCTION}

The mechanical properties and shapes of an animal cell depend largely on a protein called actin [1]. This protein can assemble into filamentous structures that form a network beneath the plasma membrane, which envelops the cell. Actin filaments are polar as their two ends are structurally different. They are referred to as the barbed or plus end and the pointed or minus end, respectively. Actin filaments often form structures like bundles and rings. Rings appear, for example, during the late phases of cell division called cytokinesis, when an actin ring cleaves the mother cell to form two daughter cells [2]. In epithelial cells, actin rings exist permanently and their coordinated contraction can induce morphological processes by bending or folding of an epithelium [3].

In cellular contractile rings, actin filaments interact via passive and active cross-linkers [1]. Passive cross-linkers, like the proteins $\alpha$-actinin or filamin, can bind simultaneously to two

\footnotetext{
*fhk@lusi.uni-sb.de

${ }^{\dagger}$ chhof@lusi.uni-sb.de

†riveline@unistra.fr

§karsten.kruse@unige.ch
}

actin filaments and influence the actin network structure as well as its mechanical properties. These cross-links have a finite life time of about one minute such that the network behaves as a fluid on long time scales [4]. Active cross-links are established by molecular motors that can transform the chemical energy released by hydrolysis of adenosine-triphosphate (ATP) into mechanical work [1]. Actin filaments interact with motors of the myosin superfamily. Notably, nonmuscle myosin II forms small aggregates that are able to cross-link actin filaments. Upon ATP hydrolysis cross-linking motor aggregates will actively generate mechanical stresses in the actin network. The orientation of the force dipoles generated by myosin II depends on the orientation of the linked filaments as the motor exerts forces into the direction of a filament's pointed end. As a consequence of the motor activity, two antiparallel actin filaments linked by a myosin aggregate will slide relative to each other until their barbed ends meet; see Fig. 1.

How cytokinetic rings of actin filaments, motors, and passive cross-linkers are organized and how they generate stresses that can lead to their contraction is still poorly understood. This is in marked contrast to myofibrils, the basic strands of striated muscle fibers. These present a periodic arrangement of interdigitating actin and myosin filaments [1]. The orientation of the filaments is such that the sarcomeres, that is, the 

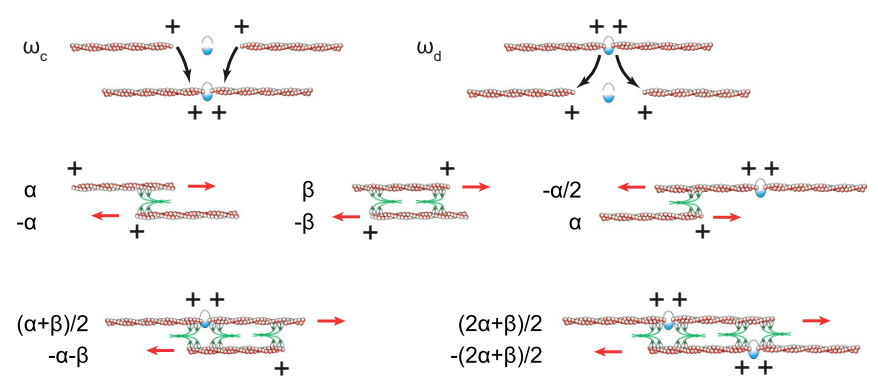

FIG. 1. Illustration of the kinetic processes captured by the dynamic Eqs. (1)-(11). Top: assembly and disassembly of bipolar filaments from polar filaments. Middle and bottom: filament displacements induced by cross-linking molecular motors. Each plus end carries a motor. Depending on the relative orientation of the linked filaments, the displacement velocity of a filament of length $\ell$ is either $\alpha$ (parallel filaments) or $\beta$ (antiparallel filaments). In the case of interactions involving bipolar filaments, the contributions of several molecular motors can add (bottom). This leads to the values of the displacement velocity that are indicated with each interacting filament pair. Red arrows indicate the direction of motion of the filaments.

elementary cells of a myofibril, readily contract upon activation of the motors. For a priori disordered filament bundles and higher dimensional networks, several mechanisms of net stress generation have been proposed [5-9]. Furthermore, reconstitution experiments in vitro have given some support for the roles of nonlinear filament elasticity [10] and local network geometry [11] for generating net contractility. Advances on the contraction mechanism for rings in live cells had been difficult to make, because cells typically divide perpendicular to the plane of observation making imaging of actimyosin rings during constriction a challenge. Using microfabricated wells, fluorescence microscopy of entire rings in a single focal plane was reported [12-14]. In these rings, myosin spontaneously formed clusters [15] that moved along the ring perimeter in fission yeast cells, but not in HeLa cells, which are taken from an immortal cell line and are commonly used as prototypic mammalian cells. The cluster motion in yeast cells presented a rich set of dynamic phenomena, namely, clockwise and counter clockwise motion as well as cluster fusion, elongation, and separation; see Fig. 2. The two different kinds of patterns were proposed to be associated with different functionalities, namely, active contraction in mammalian cells and transport in fission yeast cells.

Through a physical description of motor-filament bundles, it was shown that the patterns observed in mammalian cells and in yeast could be generated by the same mechanism [15]. In the present work, we provide a detailed analysis of the dynamic processes introduced in Ref. [15]. In our description, polar filaments represent individual actin filaments, whereas the relative sliding represents the action of molecular motors. In addition to previously studied descriptions of filament motor bundles $[5,16,17]$, we here also include bipolar filaments. There is currently no direct evidence for the presence of bioplar structures in contractile actomyosin rings. However, the myosin nodes reported in actomyosin rings in fission yeast [18] strongly suggest their presence in this system. Also, there are now a number of reports on Myosin clusters in actin networks, for example, Refs. [19-22]. These structures are associated with actin filament barbed ends in close proximity. In two and three spatial dimensions, these actomyosin structures are known as "asters," which have been observed beneath the plasma membrane [23] and, after interfering with actin filament assembly, in lamellipodia [24], which are two-dimensional cellular protrusions involved in cell migration. Confined to one spatial dimension-as is essentially the case for the actomyosin rings considered here-they can be described as bipolar filaments.

After presenting the system, we will perform a linear stability analysis of the homogenous stationary filament distribution. We will then use numerical solutions of the full dynamic equations to determine the system's phase diagram. In particular, we find solitary wave solutions that had not been shown before for this system. We also report experimental patterns of myosin clusters in fission yeasts, and we relate them to specific phases. Finally, we will discuss our results in the light of experimental observations and point out the potential value of myosin clusters for inferring actin orientations in vivo.

\section{SYSTEM AND THEORETICAL DESCRIPTION}

For our description of the filament bundles, we take an approach similar to the one developed in Ref. [5]. Filaments are assumed to be rigid rods of length $\ell$ with two distinguishable ends. Two filaments of opposite orientation can join at their plus ends and form a bipolar filament of length $2 \ell$; see Fig. 1. They can split again into two polar filaments of length $\ell$. We choose a mean-field approach in one spatial dimension for describing the dynamics of the actin filaments along the ring perimeter with coordinate $x$, where $0 \leqslant x \leqslant L$. The reduction to one spatial dimension is justified by the fact that the extension of the rings in the direction perpendicular to the circumference is much smaller than their perimeter $(200 \mathrm{~nm}$ compared to $20 \mu \mathrm{m}$ [15], Fig. 1). The state of the system is given by the distribution of the filament plus ends, which in the context of our one-dimensional description amount to the projection of the corresponding distributions in three dimensions on the ring contour. This distribution is denoted by $c^{+}$and $c^{-}$for the polar filaments of the two possible orientations and $c^{\text {bp }}$ for the bipolar filaments. Their time evolution is governed by the following continuity equations:

$$
\begin{gathered}
\partial_{t} c^{+}=D \partial_{x}^{2} c^{+}-\partial_{x} J^{+}-\omega_{c} c^{+} c^{-}+\omega_{d} c^{\mathrm{bp}}, \\
\partial_{t} c^{-}=D \partial_{x}^{2} c^{-}-\partial_{x} J^{-}-\omega_{c} c^{+} c^{-}+\omega_{d} c^{\mathrm{bp}}, \\
\partial_{t} c^{\mathrm{bp}}=D \partial_{x}^{2} c^{\mathrm{bp}}-\partial_{x} J^{\mathrm{bp}}+\omega_{c} c^{+} c^{-}-\omega_{d} c^{\mathrm{bp}} .
\end{gathered}
$$

Here, $D$ denotes the diffusion constant, $J^{ \pm}$and $J^{b p}$ are the motor-induced currents discussed in detail below, and $\omega_{d}$ and $\omega_{c}$ quantify the rate at which bipolar filaments are formed and break up, respectively. The first terms on the right-hand sides are effective diffusion terms that account for fluctuations in the system. For simplicity, we chose the three diffusion constants to be equal. If fluctuations were purely thermal, one would roughly expect a factor of 2 between the diffusion constants of polar and bipolar filaments. Here, however, fluctuations are also due to and probably dominated by the stochastic action of molecular motors and stochastic filament assembly, 


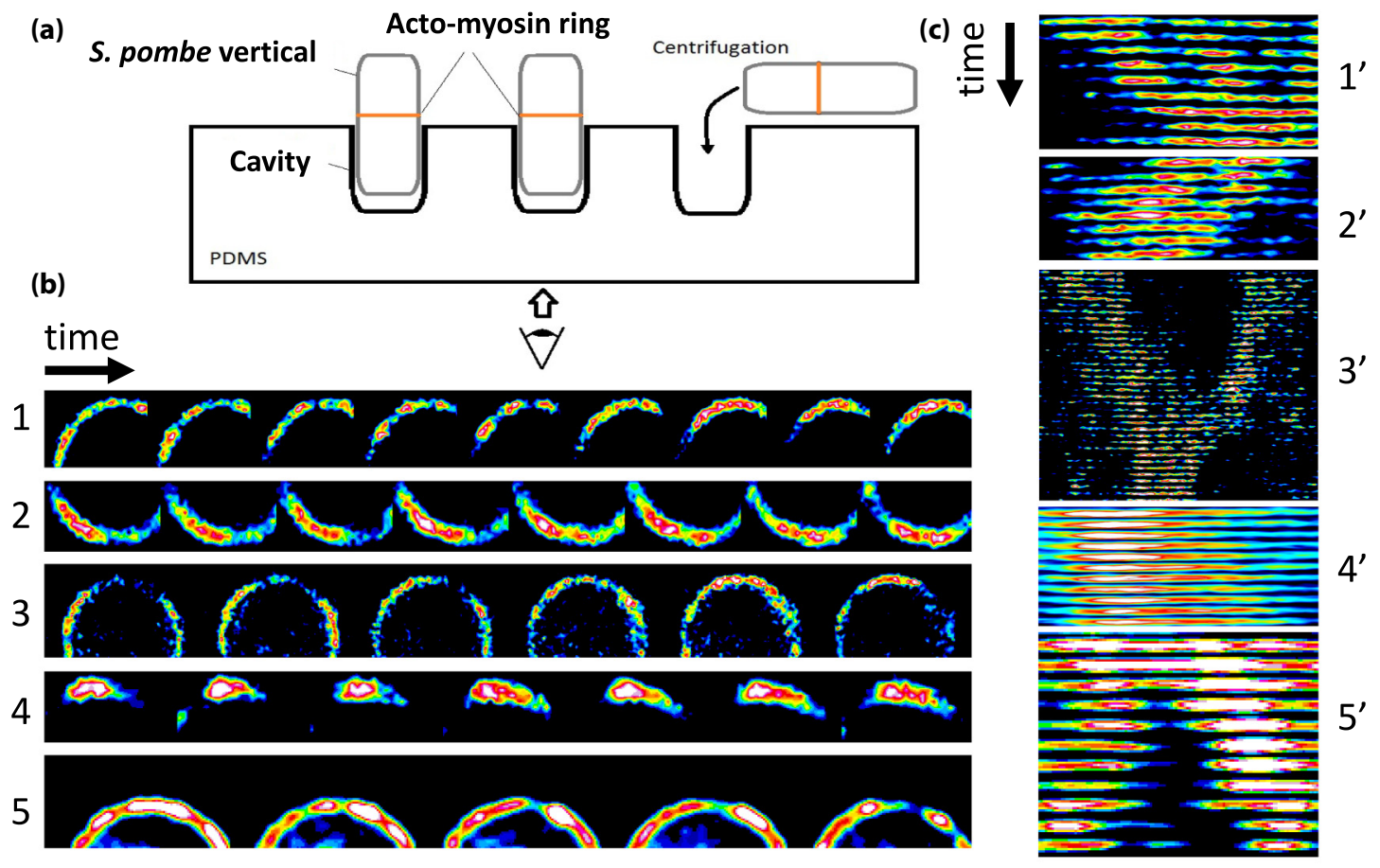

FIG. 2. Dynamics of myosin clusters within cytokinetic rings in fission yeast. (a) Experimental setup: fission yeast cells are inserted in microfabricated cavities to align the rings with the confocal plane of the microscope [12]. (b) Snapshots of the density of fluorescently labeled myosin (Rlc1) along parts of different rings [15]. Higher densities correspond to warmer colours; the look-up tables, contrast, and brightness optimize the visualization of clusters. Ring diameters are $2.2 \mu \mathrm{m}(1,2), 4.3 \mu \mathrm{m}(3), 1.9 \mu \mathrm{m}(4)$, and $1.7 \mu \mathrm{m}(5)$. Time difference between subsequent snapshots are $4 \mathrm{~s}(1,2,4), 24 \mathrm{~s}(3)$, and $8 \mathrm{~s}(5)$. Images were acquired at $27^{\circ} \mathrm{C}(1,2,4)$ and $36^{\circ} \mathrm{C}(3,5)$. (c) Kymographs corresponding to the snapshots in (b) after a polar transformation of the ring segments to a straight line. Time difference between horizontal lines: $4 \mathrm{~s}$.

which leads to a different dependence of the effective diffusion constants on the filament length. In any case, we have checked that differences in the diffusion constants have only limited effects on the system behavior.

We can estimate the value of the diffusion constant: The coefficient for myosin on actin is $\eta \sim 0.3 \mathrm{pN} \mathrm{s} \mu \mathrm{m}^{-2}$ [25]. Assuming that we can use the Stokes-Einstein relation [26], we obtain $D \sim k_{B} T \eta^{-1} a^{-1} \sim 0.1 \mu \mathrm{m}^{2} \mathrm{~s}^{-1}$, where $a=5 \mathrm{~nm}$ is the size of a motor molecule. This result is consistent with the measured value [15].

The reaction terms in Eqs. (1)-(3) describe the formation and breaking up of bipolar filaments. Formation is a process of second order with a rate constant $\omega_{c}$, whereas breaking up occurs at a rate $\omega_{d}$. The total numbers of plus and minus filaments, $N^{+}=\int_{0}^{L}\left(c^{+}+c^{\mathrm{bp}}\right) d x$ and $N^{-}=\int_{0}^{L}\left(c^{-}+c^{\mathrm{bp}}\right) d x$, are conserved by these reactions.

The remaining terms account for the effects of molecular motors. As stated in the Introduction, molecular motors can cross-link filaments and induce relative sliding. This leads to macroscopic filament currents $J^{ \pm}$and $J^{\text {bp }}$ for polar and bipolar filaments, respectively. We will assume that there is a motor molecule at each plus end. We will comment on this assumption in Sec. V. For the time being, suffice it to state that other motor distributions lead to qualitatively similar dynamics. As we will restrict attention to two-filament interactions, the filament currents can be split into three parts describing the effects of interactions between the three kinds of filaments:

$$
J^{+}=J^{++}+J^{+-}+J^{+\mathrm{bp}}
$$

$$
\begin{aligned}
& J^{-}=J^{-+}+J^{--}+J^{-\mathrm{bp}}, \\
& J^{\mathrm{bp}}=J^{\mathrm{bp}+}+J^{\mathrm{bp}-}+J^{\mathrm{bpbp}},
\end{aligned}
$$

where the second superscript serves to distinguish between the contributions of the filament current that is due to interactions with plus-, minus-, and bipolar filaments, respectively.

Due to the finite length of the filaments, there is a finite interaction range between filaments, which leads to integral expressions for the currents. Explicitly, we consider that a cross-linking motor generates a force dipole of strength $f_{p}$ if the cross-linked filaments point into the same direction and of strength $f_{a}$ if they are antiparallel. Due to the mean-field approach that we employ here, the force dipoles of different motors simply add up. If we associate a mobility $\eta$ with single filaments and half of it, $\eta / 2$, with bipolar filaments that are twice as long, then the currents are

$$
\begin{gathered}
J^{ \pm \pm}(x)=\alpha \int_{0}^{\ell} c^{ \pm}(x)\left[c^{ \pm}(x+\xi)-c^{ \pm}(x-\xi)\right] d \xi, \\
J^{ \pm \mp}(x)=\mp \beta \int_{0}^{\ell} c^{ \pm}(x) c^{\mp}(x \mp \xi) d \xi, \\
J^{ \pm \mathrm{bp}}(x)=\alpha \int_{0}^{\ell} c^{ \pm}(x)\left[c^{\mathrm{bp}}(x+\xi)-c^{\mathrm{bp}}(x-\xi)\right] d \xi \\
\mp \beta \int_{0}^{\ell} c^{ \pm}(x) c^{\mathrm{bp}}(x \mp \xi) d \xi,
\end{gathered}
$$


(a)

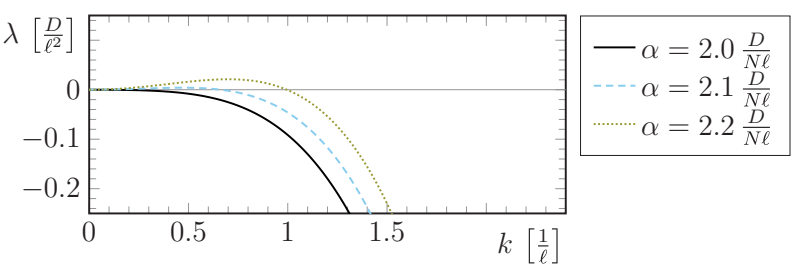

(b)

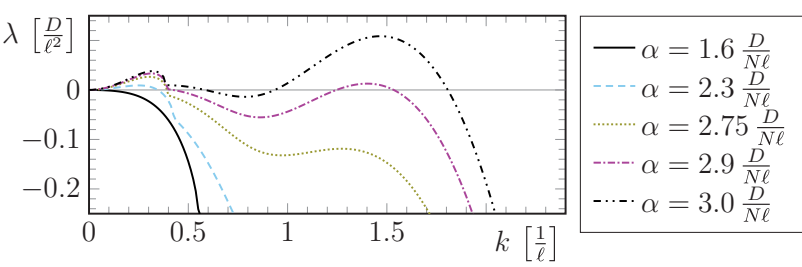

(c)

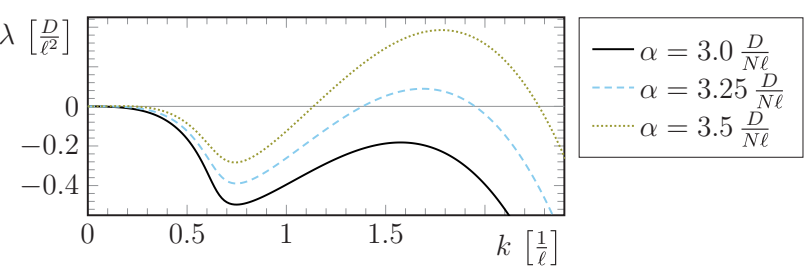

FIG. 3. Growth rate of modes from the linearized dynamic Eq. (14) as a function of the wave number $k$. (a) At the bifurcation, the smallest wave number is the most unstable for $\beta=0.5$ and $\omega_{d}=1.8$. (b) The mode with the smallest wave number is the most unstable for $\alpha=\alpha_{c}$, but separated by an interval of stable modes another interval of unstable modes exists for some larger values of $\alpha$ for $\beta=10$ and $\omega_{d}=1.2$. (c) The most unstable mode has a finite wave number for $\beta=20$ and $\omega_{d}=1.8$. Note that for systems of size $L$ only wave numbers $k_{n}=2 \pi n / L$ with $n=0,1,2, \ldots$ are permitted.

$$
\begin{gathered}
J^{\mathrm{bp} \pm}(x)=\frac{\alpha}{2} \int_{0}^{\ell} c^{\mathrm{bp}}(x)\left[c^{ \pm}(x+\xi)-c^{ \pm}(x-\xi)\right] d \xi \\
\pm \frac{\beta}{2} \int_{0}^{\ell} c^{\mathrm{bp}}(x) c^{ \pm}(x \pm \xi) d \xi \\
J^{\mathrm{bpbp}}(x)=\frac{2 \alpha+\beta}{2} \int_{0}^{\ell} c^{\mathrm{bp}}(x)\left[c^{\mathrm{bp}}(x+\xi)-c^{\mathrm{bp}}(x-\xi)\right] d \xi
\end{gathered}
$$

where $\alpha=f_{p} \eta$ and $\beta=2 f_{a} \eta$. The factor 2 appears in the expression for $\beta$, because there are always two motors linking antiparallel filaments. We choose the values of $\alpha$ and $\beta$ to be positive. As the bipolar filaments have a different mobility than the polar filaments, the total current $I=$ $\int_{0}^{L}\left[J^{+}(x)+J^{-}(x)+J^{\mathrm{bp}}(x)\right] d x$ does in general not vanish. For example, for the homogenous distribution $c^{ \pm, \mathrm{bp}}=c_{0}^{ \pm \text {,bp }}=$ const, we have $I=\beta \ell L\left(c_{0}^{-}-c_{0}^{+}\right) c_{0}^{\mathrm{bp}} / 2$, which is zero only if $c_{0}^{+}=c_{0}^{-}$unless there are no bipolar filaments or their interaction vanishes. This is reminiscent of the situation in higher dimensions, where the different mobilities in the directions parallel and perpendicular of a filament can lead to a motor-induced movement of the filament pair's center of mass [27]. It is different from the system in Ref. [5], where all filaments had the same mobility, such that the absence of external forces implied $I=0$. The system specification is completed by imposing periodic boundary conditions, which reflect the annular topology of actomyosin rings.
The above equations differ to some extent from the ones given in Ref. [15]. The reason is that in Ref. [15] the forces of motors at the center of a bipolar filament were treated differently from the present work. Note also that here we do not include a current resulting from filament treadmilling as was done in Ref. [15]. Such a current accounts for the displacements of filaments due to addition of monomers at one and removal of monomers at the other end [28]. For the reader's convenience, we reproduce the dynamic equations from Ref. [15] and discuss the differences to those used in the present work in the Appendix. In spite of these differences, the main states reported in Ref. [15] are also produced by the present equations and the topology of the phase space, too, is largely conserved between the two systems. For $\omega_{c}=0$, the system is essentially the same as the one introduced in Ref. [5]. ${ }^{1}$ Note, however, that the presence of bipolar filaments is essential for reproducing the experimentally observed patterns as we will see below.

In the following, we will use dimensionless parameters. Scaling all lengths by the filament length $\ell$, time by $\ell^{2} / D$, and the densities by $N / \ell$, where $N=N^{+}+N^{-}$denotes the total number of filaments, leaves us with five independent dimensionless parameters: the interaction strengths $\alpha \ell N / D$ and $\beta \ell N / D$, the rate $\omega_{d} \ell^{2} / D$, and the constant $\ell \omega_{c} N / D$, which determine the formation and breakup of bipolar filaments, and the system size $L / \ell$. We will keep the same notation for the parameters and fields as before. It turns out that changing $\omega_{c}$ has similar but opposite effects to changing $\omega_{d}$, such that we will keep $\omega_{c}=0.1$ in the remainder of this work.

\section{LINEAR STABILITY ANALYSIS}

As mentioned in the Introduction, the nascent actomyosin ring in mammalian cells is homogenous [15]. We will thus start the analysis of the above equations by determining the linear stability of the homogenous stationary state. In the homogenous state, the divergences of all currents vanish. Note that although the individual currents might be different from zero, the total net current vanishes. It is stationary if the homogenous densities $c_{0}^{ \pm}$and $c_{0}^{\mathrm{bp}}$ satisfy $\omega_{c} c_{0}^{+} c_{0}^{-}=\omega_{d} c_{0}^{\mathrm{bp}}$. These densities are determined once the fractions, $n^{+} \equiv N^{+} / N$ and $n^{-} \equiv N^{-} / N$ of plus and minus filaments, respectively, are given. Indeed, $c_{0}^{-}$and $c_{0}^{\text {bp }}$ can be expressed in terms of $c_{0}^{+}$: $c_{0}^{\mathrm{bp}}=n^{+} / L-c_{0}^{+}$and $c_{0}^{-}=\left(n^{-}-n^{+}\right) / L+c_{0}^{+}$. In turn, the concentration $c_{0}^{+}$is determined from the above equation, which has one positive solution. Therefore, there is a unique stationary homogenous state. In the following, we will focus attention to the symmetric case $n^{+}=n^{-}=1 / 2$. In that case,

$$
c_{0}^{ \pm}=\frac{\omega_{d}}{2 \omega_{c}}\left[\sqrt{1+\frac{2}{L} \frac{\omega_{c}}{\omega_{d}}}-1\right] .
$$

Consider a (small) perturbation $\delta c^{ \pm \text {,bp }}(x)$ of the stationary homogenous densities. Writing $c^{ \pm}(x)=c_{0}^{ \pm}+\delta c^{ \pm}(x)$ and

\footnotetext{
${ }^{1}$ Since we specifically consider the motors to be attached to filament plus ends, however, the integrals in the expressions for $J^{ \pm \mp}$ only extend from 0 to $\ell$ and not from $-\ell$ to $\ell$ as in the previous work.
} 
(a)

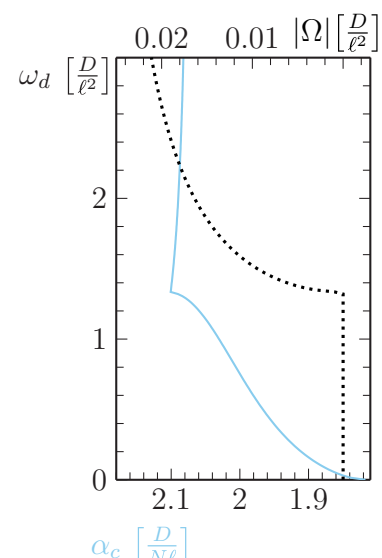

(c)
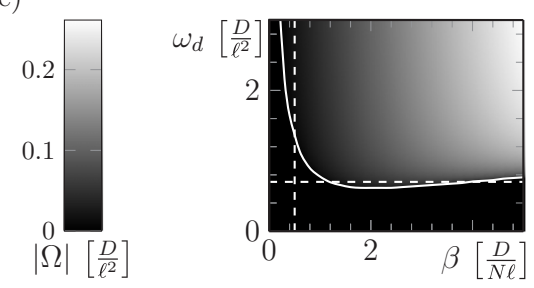

(b)

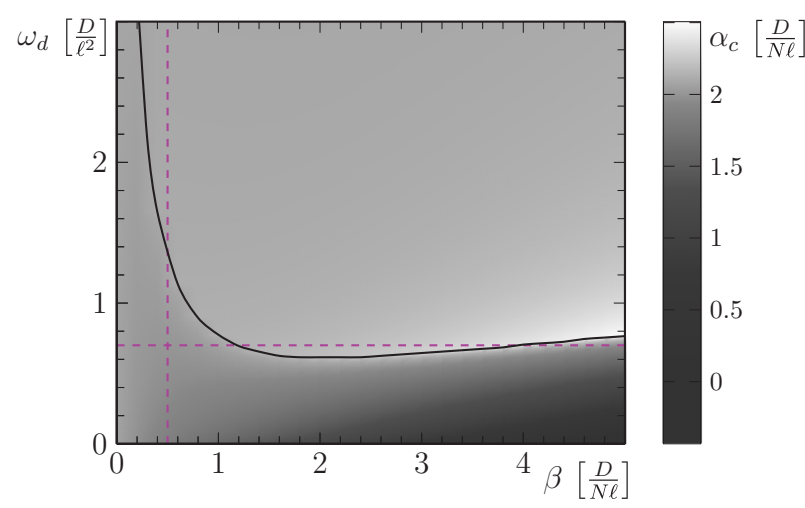

(d)

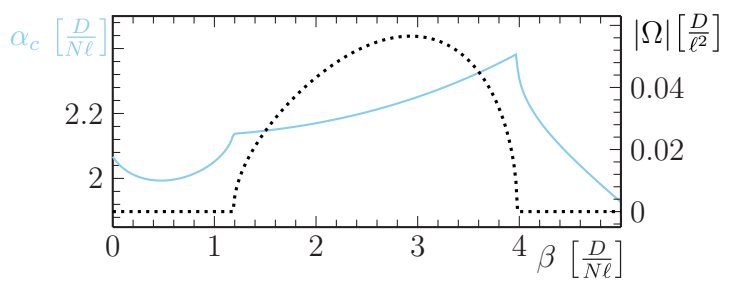

FIG. 4. Critical value $\alpha_{c}$ as a function of the interaction strength $\beta$ and the dissociation rate of bipolar filaments $\omega_{d}$. The homogenous state is stable for $\alpha<\alpha_{c}$. (a) The critical value as a function of the dissociation rate $\omega_{d}$ for an interaction strength $\beta=0.5$. (b) The critical value as a function of $\beta$ and $\omega_{d}$. The solid line separates the region, where the critical eigenvalue has a vanishing imaginary part (below the line) from the region, where the imaginary part does not vanish (above the line). The dashed lines indicate the cuts presented in (a) and (d). (c) Imaginary part of the critical growth exponent as a function of $\beta$ and $\omega_{d}$. The dashed and solid lines have the same meaning as in (b). (d) The critical value as a function of $\beta$ for $\omega_{d}=0.75$. System size $L=10 \ell$.

$c^{\mathrm{bp}}(x)=c_{0}^{\mathrm{bp}}+\delta c^{\mathrm{bp}}(x)$, we linearize the dynamic Eqs. (1)(11) in the perturbations. It is helpful to expand the perturbations in terms of a Fourier series

$$
\delta c^{ \pm, \mathrm{bp}}=\sum_{k \in k_{0} \mathbb{Z}} \hat{c}_{k}^{ \pm, \mathrm{bp}} \exp (\mathfrak{i} k x),
$$

where $k_{0}=\frac{2 \pi}{L}$ denotes the wave number of the fundamental mode of the bundle. The linearized dynamic equations then read

$$
\frac{d}{d t}\left(\begin{array}{c}
\hat{c}_{k}^{+} \\
\hat{c}_{k}^{-} \\
\hat{c}_{k}^{\mathrm{bp}}
\end{array}\right)=\left(\begin{array}{ccc}
\Lambda_{k}^{++} & \Lambda_{k}^{+-} & \Lambda_{k}^{+\mathrm{bp}} \\
\Lambda_{k}^{-+} & \Lambda_{k}^{--} & \Lambda_{k}^{-\mathrm{bp}} \\
\Lambda_{k}^{\mathrm{bp}+} & \Lambda_{k}^{\mathrm{bp}-} & \Lambda_{k}^{\mathrm{bpbp}}
\end{array}\right)\left(\begin{array}{c}
\hat{c}_{k}^{+} \\
\hat{c}_{k}^{-} \\
\hat{c}_{k}^{\mathrm{bp}}
\end{array}\right)
$$

where the matrix components are given by

$\Lambda_{k}^{ \pm \pm}=-\omega_{c} c_{0}^{\mp}-k^{2} \pm \mathfrak{i} \beta k\left(c_{0}^{\mp}+c_{0}^{\mathrm{bp}}\right)-2 \alpha[\cos (k)-1] c_{0}^{ \pm}$,

$$
\begin{gathered}
\Lambda_{k}^{ \pm \mp}=-\omega_{c} c_{0}^{ \pm}+\beta\left[1-e^{\mp \mathrm{i} k}\right] c_{0}^{ \pm}, \\
\Lambda_{k}^{ \pm \mathrm{bp}}=\omega_{d}-2 \alpha[\cos (k)-1] c_{0}^{ \pm}-\beta\left[e^{\mp \mathrm{i} k}-1\right] c_{0}^{ \pm}, \\
\Lambda_{k}^{\mathrm{bp} \pm}=\omega_{c} c_{0}^{\mp}-\alpha[\cos (k)-1] c_{0}^{\mathrm{bp}}+\frac{\beta}{2}\left[e^{ \pm \mathrm{i} k}-1\right] c_{0}^{\mathrm{bp}}, \\
\Lambda_{k}^{\mathrm{bpbp}}=-\omega_{d}-k^{2}-\mathfrak{i} \frac{\beta}{2} k\left(c_{0}^{+}-c_{0}^{-}\right) \\
-(2 \alpha+\beta)[\cos (k)-1] c_{0}^{\mathrm{bp}} .
\end{gathered}
$$

For each wave number $k$, we then have three modes that are given by the eigenvectors of this matrix. The corresponding eigenvalues determine their stability: if the eigenvalue has a negative real part, then the mode will relax and the homogenous state is linearly stable against perturbations by this mode. If it has a positive real part, then the mode will grow and the homogenous state is unstable. There are critical surfaces in parameter space that separate regions in which all modes are stable from regions where at least one mode is unstable. To facilitate comparison with earlier works, we will discuss these boundaries in terms of critical values $\alpha_{c}$ of the interaction strength $\alpha$.

First, let us note that for $k=0$, one eigenvalue of the matrix in Eq. (14) is zero, whereas the other two have a negative real part. The homogenous state is thus stable against homogenous perturbations. The dispersion relation, which gives the growth rate as a function of the wave number $k$, is shown for a few parameter values in Fig. 3. In large regions of parameter space, we found the mode with the smallest wave number to be the first to become unstable as the interaction strength $\alpha$ is increased; see Fig. 3(a). As the interaction strength $\alpha$ is increased beyond its critical vale $\alpha_{c}$ an interval of unstable modes extends from $k=0$. For some regions in parameter space, a second interval of unstable modes appears as $\alpha$ is increased further; see Fig. 3(b). For small enough systems, the fundamental wave number $k_{0}=2 \pi / L$ can fall into the interval of stable modes between the two intervals of unstable modes and thus a mode with $k>k_{0}$ can be unstable at the critical value $\alpha_{c}$. In still other cases, there are no unstable modes in an interval extending from $k=0$; see Fig. 3(c), such that a mode with 
(a)

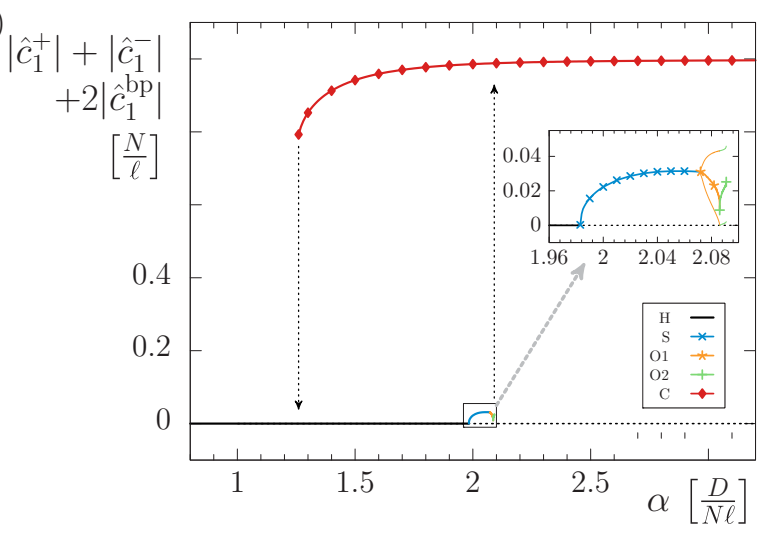

(b)

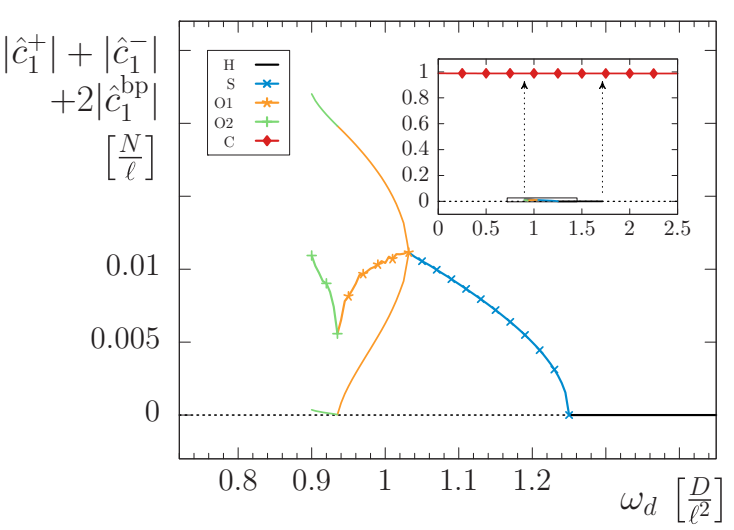

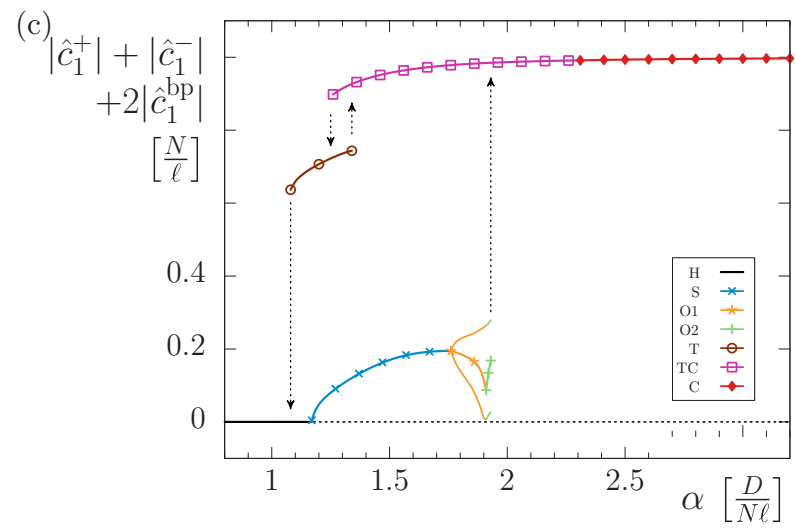

FIG. 5. Bifurcation diagrams. Asymptotic states are represented by the sums of the moduli of the first Fourier modes of $c^{ \pm}$and $c^{\text {bp }}$. For the oscillatory states, the mean as well as the minimal and maximal values are presented. (a) $\beta=0.5$ and $\omega_{d}=0.5$, (b) $\beta=0.5$ and $\alpha=2.11$, (c) $\beta=10$ and $\omega_{d}=0.8$. In all cases $\omega_{c}=0.1$ and $L=10$.

$k>k_{0}$ can be the most unstable one. Such dispersion relations are indicative of the spontaneous emergence of patterns with an intrinsic length scale for $\alpha>\alpha_{c}$.

We now turn to the dependence of $\alpha_{c}$ on the parameters $\beta$ and $\omega_{d}$. The effect of the system length $L$ will be briefly discussed below. In Fig. 4, we present $\alpha_{c}$ as a function of $\beta$ and $\omega_{d}$. The homogenous state is stable for $\alpha<\alpha_{c}$ and unstable for $\alpha>\alpha_{c}$. Two different regimes that are separated by a "ridge" in the $\alpha_{c}$ landscape can be distinguished. They correspond to different behaviors at the stability threshold. For $\omega_{d}<\omega_{d}^{r}$, where $\omega_{d}^{r}(\beta)$ marks the position of the ridge, the imaginary part of the critical eigenvalue vanishes. This is indicative of a stationary state beyond the instability. In contrast, for $\omega_{d}>\omega_{d}^{r}$, the imaginary part does not vanish, which suggests an oscillatory state beyond the instability. With increasing values of $\omega_{d}$, the value of $\alpha_{c}$ first increases and then decreases again; see Fig. 4. The maximum marks the frontier between real and complex critical eigenvalues. This picture is essentially the same as the one reported in Ref. [15].

For $\omega_{d}=0.75$ the critical value $\alpha_{c}$ displays two ridges that are characterized by a discontinuity in the derivatives. Only one of the ridges coincides with a maximum of $\alpha_{c}$, Fig. 4 (bottom). Within the different intervals separated by the intersections, the critical value is a convex function of the parameter $\beta$.

The instability of the homogenous state is driven by the interactions of filaments of the same polarity, but also by the interactions between polar and bipolar filaments. This can be seen by performing the stability analysis for each of the currents Eqs. (7)-(11) individually. Since the interactions between bipolar filaments and any other filament type depend on $\beta$, the homogenous state is unstable for all $\alpha \geqslant 0$ as long as $\beta$ is sufficiently large; see Fig. 13(b). This is in contrast to the case of bundles of only polar filaments, where the critical interaction strength between filaments of the same orientation is always larger than zero [5,17].

\section{NONLINEAR DYNAMICS}

In this section, we discuss the asymptotic solutions beyond the instability of the homogenous state. They were obtained

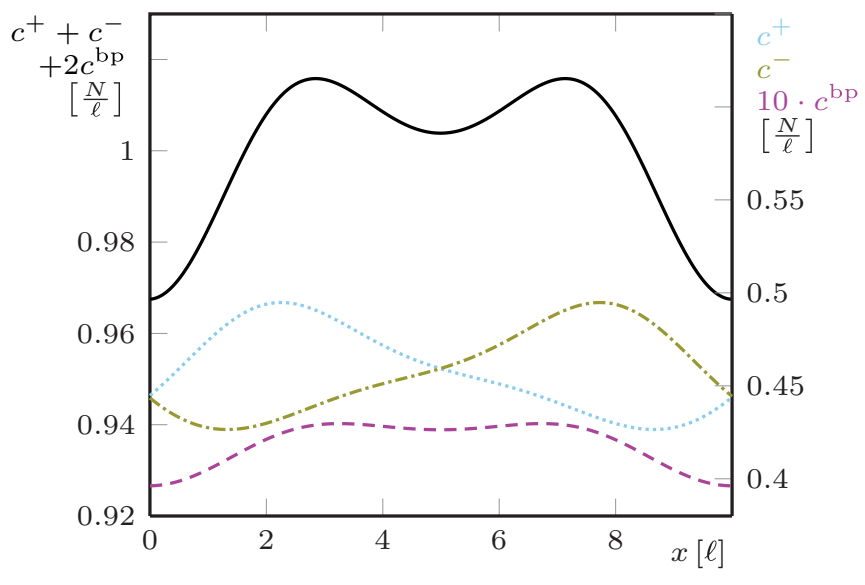

FIG. 6. Asymptotic stationary state for $\alpha=2.06, \beta=$ $0.5, \omega_{d}=0.5, \omega_{c}=0.1$, and $L=10$. 

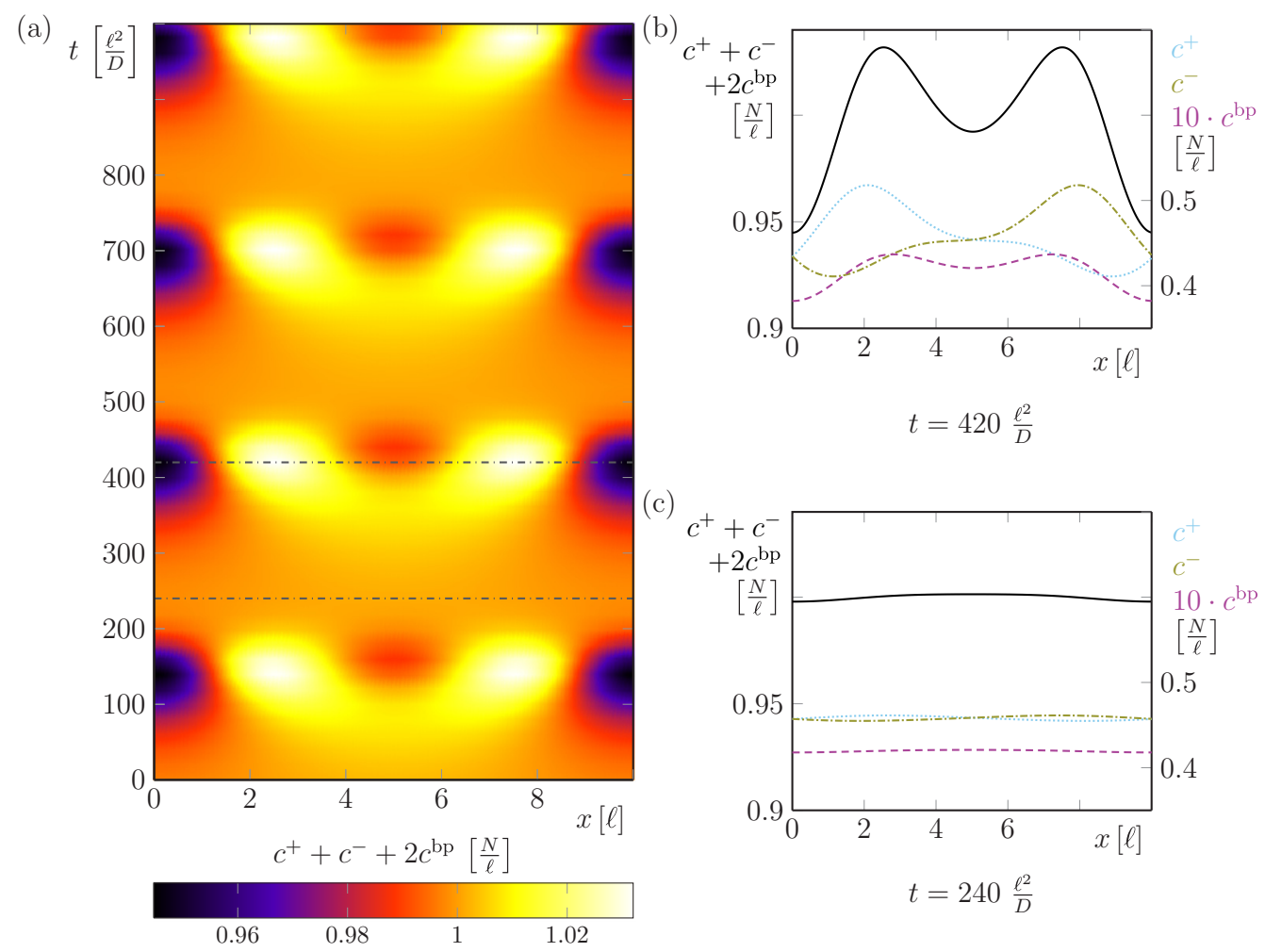

FIG. 7. Asymptotic oscillatory state $1(\mathrm{O} 1)$ for $\alpha=2.085, \beta=0.5, \omega_{d}=0.5, \omega_{c}=0.1$, and $L=10$. (a) Kymograph of the time evolution. (b, c) Density profiles for $t=420 \frac{\ell^{2}}{D}$ and $t=240 \frac{\ell^{2}}{D}$, respectively.

by numerically solving the dynamic Eqs. (1)-(11). We used a finite difference upwind scheme to discretize the right hand sides of Eqs. (1)-(3). The ensuing system of ordinary differential equations was solved numerically by an explicit Euler scheme with adaptive time steps.

\section{A. Bifurcation diagrams and nonlinear states}

Let us start by discussing the asymptotic states as the value of $\alpha$ is varied, whereas $\beta=0.5$ and $\omega_{d}=0.5$ are fixed. Consequently, $\omega_{d}<\omega_{d}^{r}$ and the critical eigenvalue is real. In that case, the homogenous state loses its stability through a supercritical forward bifurcation at $\alpha_{c}$, such that the new state emerges with vanishing amplitude; see Fig. 5(a). The nonlinear state is stationary (S) and consists mostly of polar filaments; see Fig. 6. The fraction of bipolar filaments is below $10 \%$. The total density of filament plus ends, $c^{+}+c^{-}+2 c^{\mathrm{bp}}$, has two maxima.

As indicated by the linear stability analysis we have not been able to detect a wavelength intrinsic to the pattern, such that the size of this cluster is determined by the system size. Its amplitude saturates with increasing $\alpha$, see Fig. 5(a), and subsequently the state undergoes a secondary bifurcation into an oscillatory state (O1); see Fig. 5(a). This state is a standing wave; see Fig. 7. In this state, the myosin cluster emerges, then essentially vanishes, and reemerges at the same position as before.

This state then undergoes a transition into another state (O2); see Fig. 8. The transition is evident from the bifurcation diagram even without looking at the corresponding filament distributions: the average amplitude has a kink as a function of $\alpha$; see Fig. 5(a). $\mathrm{O} 2$ is still a standing wave, but the peaks of the plus- and minus-filament distributions now periodically exchange positions.

We can get further insight into the transition between these two states by looking at the complex amplitudes of the lowest two Fourier modes. In Figs. 9(a) and 9(b), we display the orbits of the Fourier modes $\hat{c}_{1}^{ \pm}$and $\hat{c}_{1}^{\text {bp }}$ for the densities of polar and bipolar filaments. We have chosen the phase such that $\hat{c}_{1}^{\mathrm{bp}}$ is purely imaginary. It lies on the symmetry axis for $\hat{c}_{1}^{+}$and $\hat{c}_{1}^{-}$. The orbits are limit cycles corresponding to the temporal oscillation of the patterns. For $\mathrm{O} 1$ all modes have a negative imaginary part, whereas for $\mathrm{O} 2$ they exhibit a point symmetry with respect to the origin. There is no corresponding change in the second Fourier mode; see Figs. 9(c) and 9(d). The change in the orbit of the first Fourier mode of $c^{+}$suggests a transition between the $\mathrm{O} 1$ and $\mathrm{O} 2$ akin to an infinite period bifurcation, where two limit cycles fuse in a homoclinic point; see Fig. 9(e). Indeed the temporal periods show the characteristic divergence as the critical value of $\alpha$ is approached; see Fig. 9(f). Note, that for the same deviation from the critical interaction strength, the periods of the two oscillatory states differ by a factor of 2 .

The two oscillatory states extend only for a comparatively small interval of $\alpha$ values; see Fig. 5(a). When O2 loses stability, it gives way to a cluster state (C); see Fig. 10. In this state, there is again one myosin cluster. But now, the distribution of plus ends is much narrower than for the stationary state $\mathrm{S}$. It emerges through a subcritical bifurcation and coexists in a sizable interval of $\alpha$ values with the homogenous state and also with all other states discussed so far; see Fig. 5(a). The bifurcation diagram suggests that there is an unstable state 
(a)

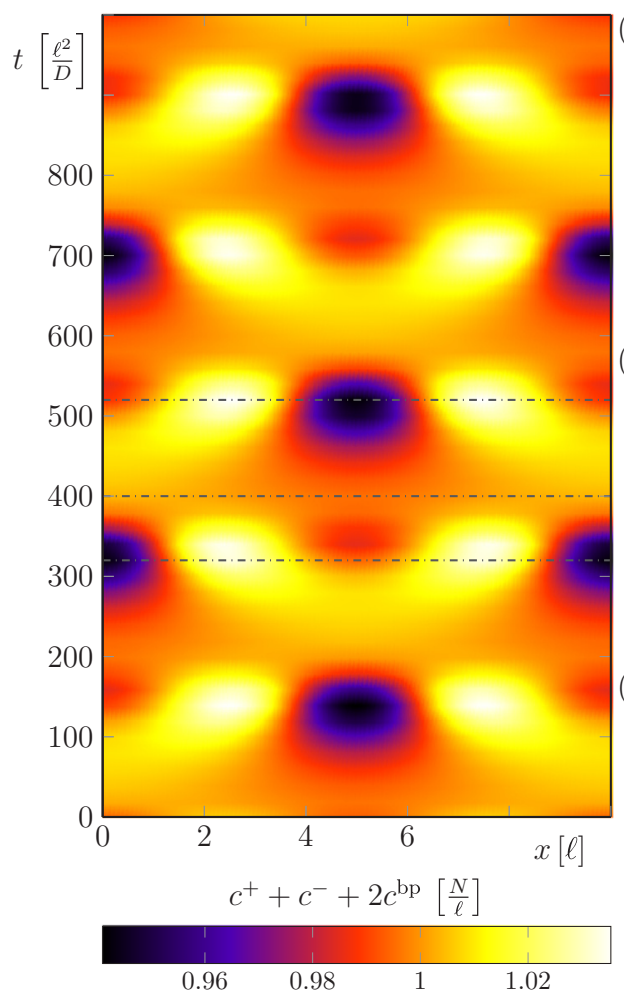

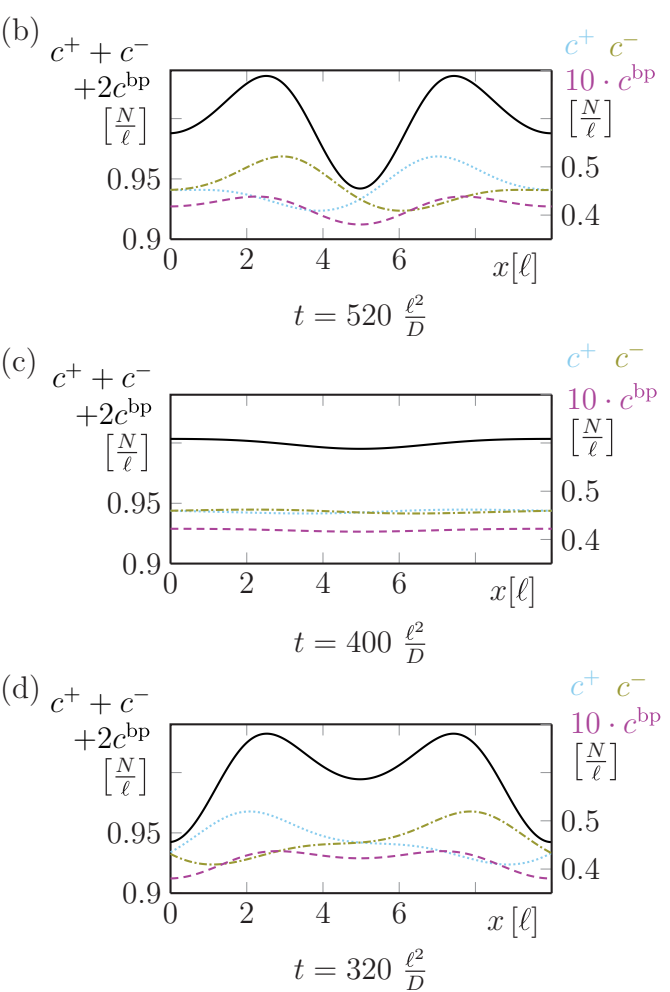

FIG. 8. Asymptotic oscillatory state 2 (O2) for $\alpha=2.09, \beta=0.5, \omega_{d}=0.5, \omega_{c}=0.1$, and $L=10$. (a) Kymograph of the time evolution. (b-d) Density profiles for $t=520 \frac{\ell^{2}}{D}, t=400 \frac{\ell^{2}}{D}$, and $t=320 \frac{\ell^{2}}{D}$, respectively.

linking the cluster state $\mathrm{C}$ to $\mathrm{O} 2$, but we have not tried to determine unstable states.

In Fig. 5(b), we present the bifurcation diagram when varying the dissociation rate of bipolar filaments. Decreasing the value of $\omega_{d}$ has a similar effect as increasing $\alpha$ : The homogenous state is stable for $\omega_{d} \geqslant \omega_{d, c}$, where $\omega_{d, c}$ is a positive critical dissociation rate. For lower values, the stationary $\mathrm{S}$ appears. Lowering the dissociation rate further, this state bifurcates into $\mathrm{O} 1$, which subsequently bifurcates into $\mathrm{O} 2$. The transition between the two standing waves occurs along the same lines as described above. For the values of $\alpha$ and $\beta$ chosen in Fig. 5(b), the cluster state $\mathrm{C}$ exists for all values of $\omega_{d}$ shown.

For larger values of $\omega_{d}$ or $\beta$ two more states than discussed so far can emerge. In Fig. 5(c), we show the bifurcation diagram as a function of $\alpha$ for $\beta=10$ and $\omega_{d}=0.8$. At the critical value $\alpha_{c}$, the homogenous state bifurcates into the stationary state $\mathrm{S}$, which in turn turns into $\mathrm{O} 1$ and then $\mathrm{O} 2$. However, the cluster state $\mathrm{C}$ is preceded by two states that exhibit traveling waves. For values of $\alpha$ below $\alpha_{c}$, we first find a subcritical bifurcation towards a traveling wave state T; see Fig. 11. A symmetric state with the profiles of $c^{+}$and $c^{-}$exchanged and moving into the opposite direction coexists with the one shown.

Through a subcritical bifurcation, the traveling wave $\mathrm{T}$ loses stability in favor of a state with two counterpropagating traveling clusters (TC); see Fig. 12. To understand this behavior, let us first recall that there are counterpropagating peaks of plus and minus filaments also in absence of bipolar filaments, $\omega_{c}=0[16,17]$. To give a qualitative understanding, note that the interactions between filaments of the same polarity lead to the formation of peaked distributions whereas the interactions between filaments of opposite polarity set them into relative motion. Because the distributions of plus and minus filaments are strongly localized, the relative motion is very small as soon as the peaks are sufficiently far apart. It is still finite, though, and the peaks will periodically travel around the ring. For the parameter values of Fig. 12, the situation is different, because bipolar filaments appear, when the peaks of polar filaments are close enough. If, during such an encounter, a sufficient number of bipolar filaments are formed, then they will keep the plus and minus filaments from veering away from each other, hence the transition to the cluster state C; see Fig. 5(c).

Let us point out that the corresponding state in Ref. [15] presented also a stationary cluster. There, this state is obtained, when the rate $\omega_{d}$ is small enough compared to the temporal period associated with TC, such that the cluster of bipolar filaments persists for a large part of the period.

\section{B. Phase diagram}

From the bifurcation diagrams, we can build phase diagrams. Figure 13 presents cuts through the phase diagrams along the $\left(\alpha, \omega_{d}\right)$-plane for two different values of $\beta$. For $\beta=0.5, \omega_{d}<\omega_{d}^{r}$, and increasing $\alpha$, the homogenous state loses stability in favor of the stationary state as described above; see Fig. 13(a). Further increase of $\alpha$ leads to the sequence of bifurcations as depicted in Fig. 5(a). The cluster state C exists and is stable for all values of $\alpha$ shown. The five different phases seemingly meet at the ridge. However, our numerics is not accurate enough to check this hypothesis thoroughly. For 
(a)

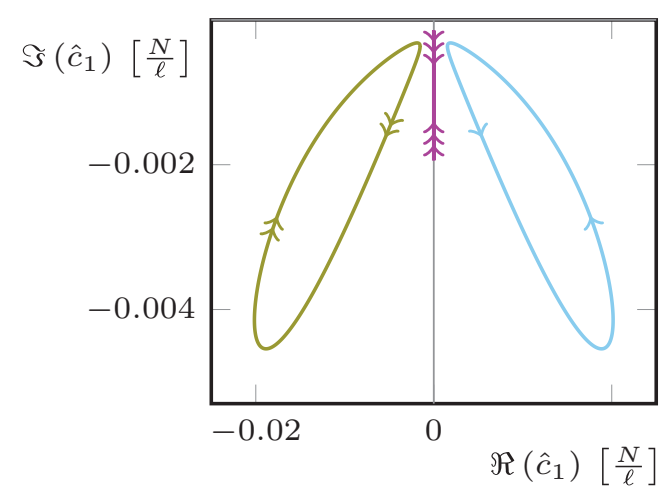

(c)

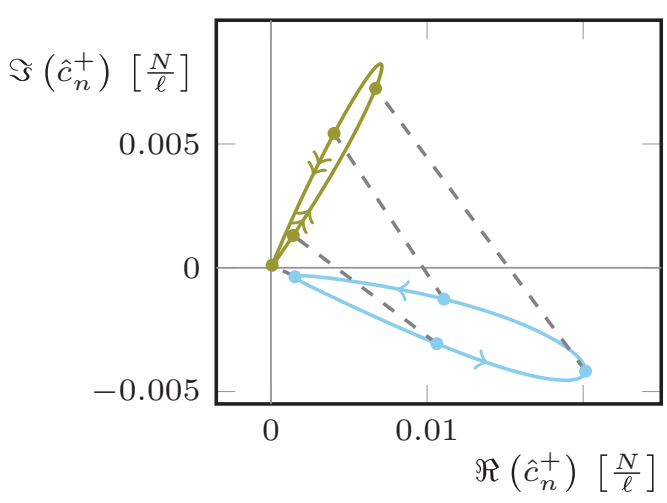

(e)

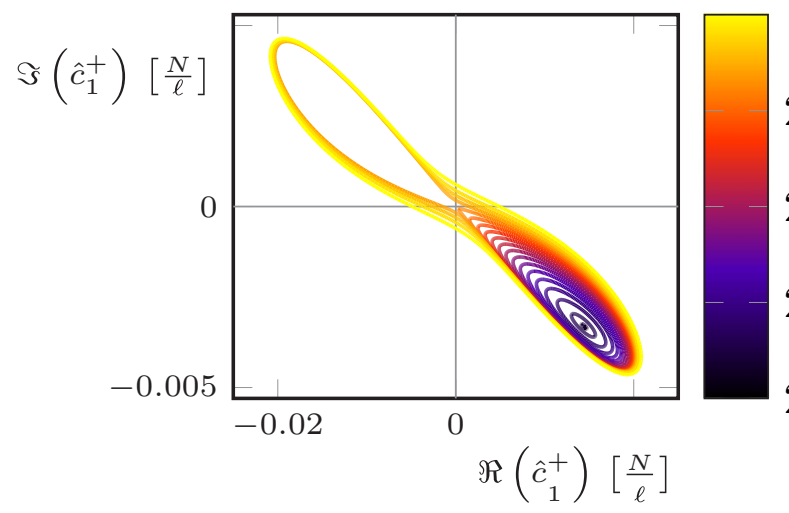

(b)

$\mathrm{O} 2$

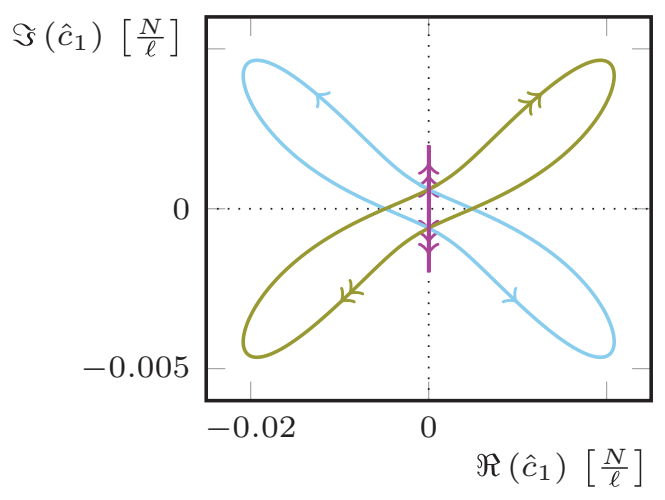

(d)

$\mathrm{O} 2$

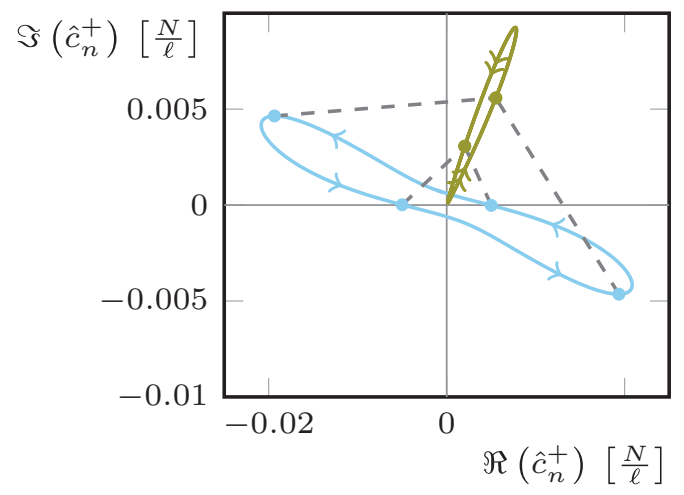

(f)

$\alpha\left[\frac{D}{N \ell}\right]$

2.085

2.08

2.075

2.07

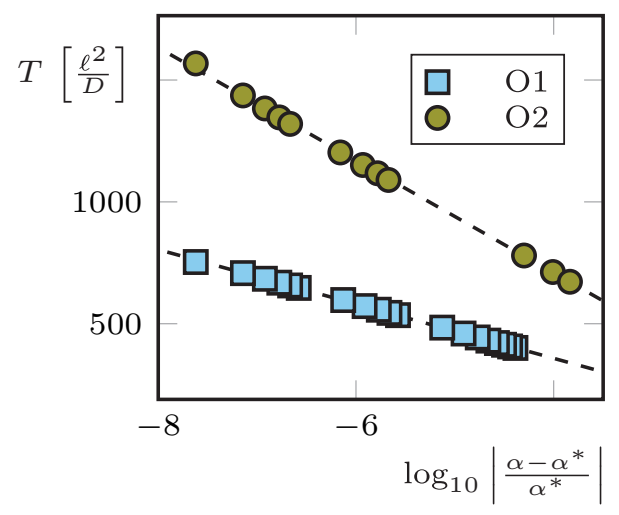

FIG. 9. Transition between $\mathrm{O} 1$ and O2. (a, b) Orbits of the amplitudes of the first spatial Fourier modes of O1 (a) and O2 (b) for $c^{+}$(blue, one arrow head), $c^{-}$(green, two arrow heads), and $c^{\text {bp }}$ (red, three arrow heads). (c, d) Orbits of the first (blue, one arrow head) and second (green, two arrow heads) spatial Fourier modes of $\mathrm{O} 1$ (c) and $\mathrm{O} 2$ (d). Dashed lines link points at equal time points. (e) Orbits of the amplitude of the first Fourier mode for $c^{+}$for different values of $\alpha$. (f) Temporal period of O1 (blue squares) and O2 (green circles) as a function of $\alpha$. Parameter values are $\alpha=2.085$ for $\mathrm{O} 1$ and $\alpha=2.09$ for $\mathrm{O} 2$ in $(\mathrm{a}-\mathrm{d})$ as well as $\beta=0.5, \omega_{d}=0.5$, and $L=10$.

smaller values of $\alpha$ than shown in the figure, the phases B-D eventually vanish. Indeed, $\alpha_{c}\left(\omega_{d}=0\right) \approx 1.82$.

For $\beta=10$, the phase diagram is much more involved; see Fig. 13(b). We will restrict ourselves to highlight only a few features. First, for sufficiently small values of $\omega_{d}$, the homogenous state is unstable even for $\alpha=0$. The interaction strength $\beta$ is large enough to induce an instability even in absence of interactions between parallel filaments. Furthermore, now also traveling waves $\mathrm{T}$ and counterpropagating clusters TC exist.
For a large interval of $\omega_{d}$, the boundary of phases containing $\mathrm{T}$ is independent of $\omega_{d}$. Maybe the most remarkable feature is that there is no bifurcation between the phases $\mathrm{E}$ (only the cluster state $\mathrm{C}$ is stable) and $\mathrm{K}$ (only the stationary state $\mathrm{S}$ is stable). Instead, the stationary state $\mathrm{S}$ transforms continuously into the cluster state C. As can be read off from Table I, in many places multiple stable states coexist. For these parameter values, the final state of the system is determined by the initial condition. 
(a)

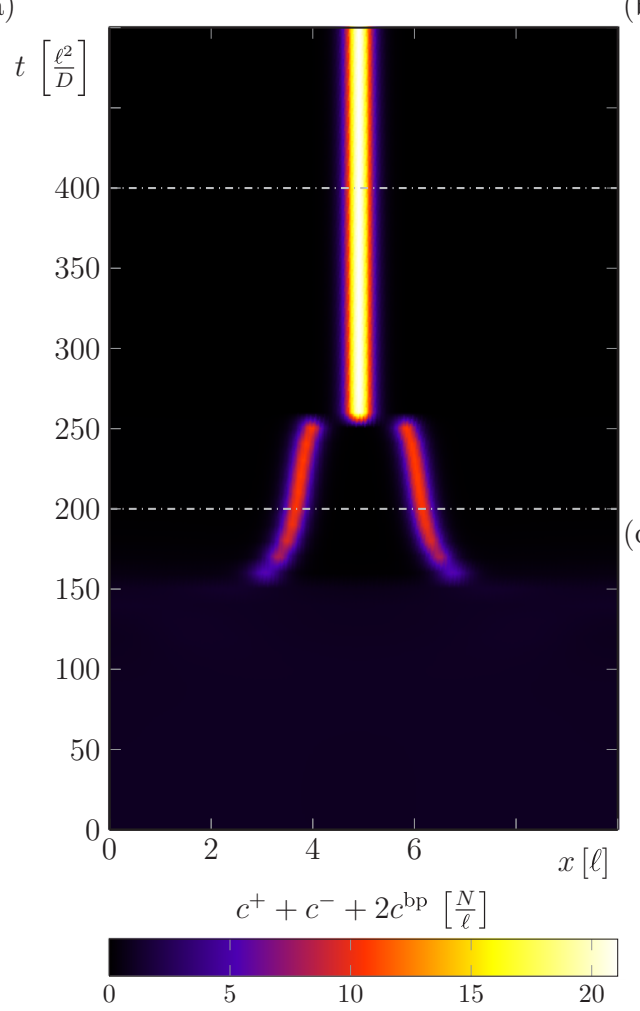

(b)
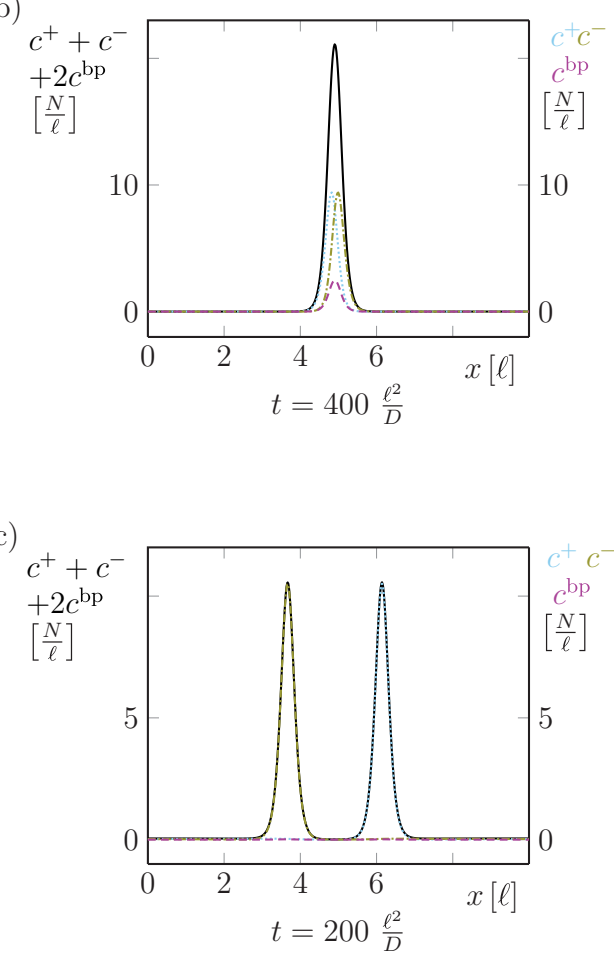

FIG. 10. Asymptotic cluster state (C) for $\alpha=2.09, \beta=0.5, \omega_{d}=0.5, \omega_{c}=0.1$, and $L=10$. (a) Kymograph for the evolution of an initially homogenous distribution with a $1 \%$ random perturbation. (b, c) Density profiles for $t=400 \frac{\ell^{2}}{D}$ and $t=200 \frac{\ell^{2}}{D}$, respectively.

\section{DISCUSSION}

In this work, we have analyzed in detail the model of actomyosin bundles introduced in Ref. [15]. It is based on pairwise interactions of polar and bipolar filaments by molecular motors. The bipolar filaments are formed by pairs of polar filaments of opposite orientation and have a finite lifetime. The presence of bipolar filaments is the most prominent difference to previous theoretical studies of motor-filament bundles. Furthermore, we assumed that the molecular motors

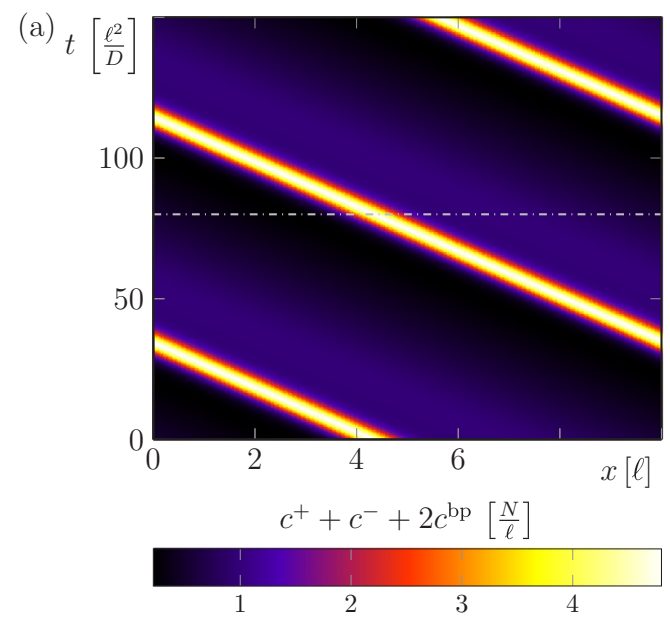

are associated with the filament plus ends. The strength of the motor-mediated interactions between filament pairs depends on the relative orientation of the two connected filaments. This is slightly different from the approach in Ref. [15]. The overall structure of the phase diagram, however, is the same and essentially also the same states are present in both models. Quantitatively, the critical value of the interaction strength $\alpha$ is smaller in the present work than in Ref. [15] and can even become negative, which implies that the homogenous state is unstable even for $\alpha=0$.

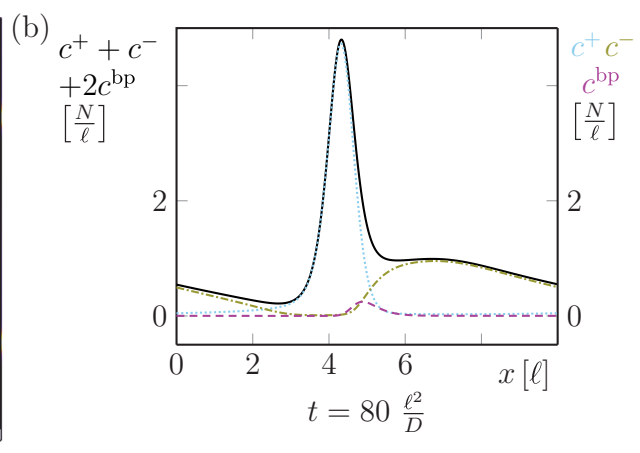

FIG. 11. Asymptotic traveling wave state (T) for $\alpha=1.12, \beta=0.5, \omega_{d}=1.8, \omega_{c}=0.1$, and $L=10$. (a) Kymograph of the time evolution. (b) Density profiles for $t=80 \frac{\ell^{2}}{D}$. 

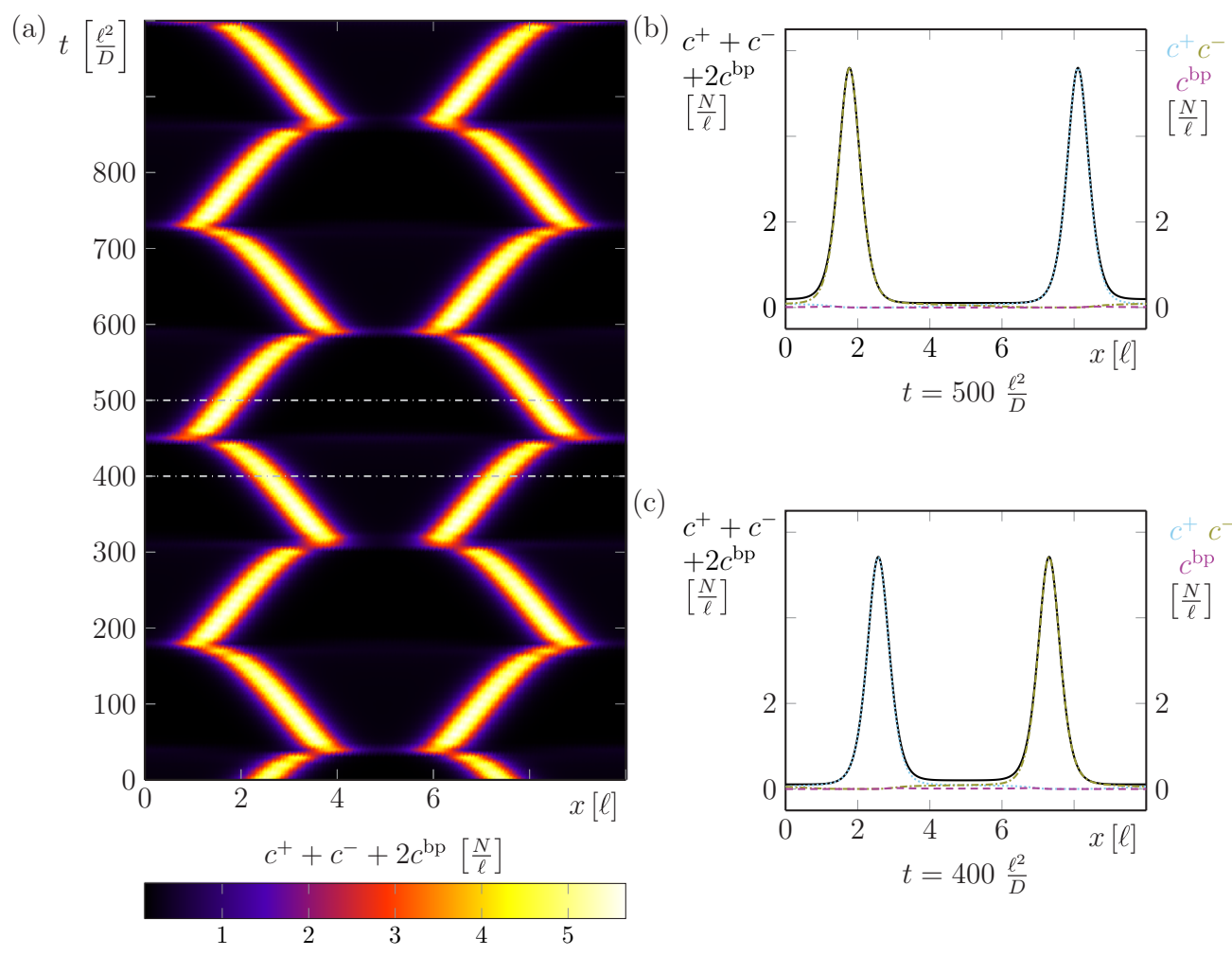

FIG. 12. Asymptotic counterpropagating traveling clusters state Kymograph of the time evolution. (b, c) Density profiles for $t=500 \frac{\ell^{2}}{D}$ and

Another difference with respect to the system introduced in Ref. [15] is that here we neglected any effects of filament assembly. In the prior work, a current due to filament treadmilling had been considered. As we have seen here, this current does not introduce qualitatively new states. It can change some aspects of the asymptotic solutions, though. For example, in Ref. [15], the state of counterpropagating clusters presented a clearly visible stationary cluster, whereas we have so far only observed comparatively small stationary clusters for TC solutions. Furthermore, the clusters of polar filaments moved in the opposite direction of what we found in the present
(TC) for $\alpha=1.28, \beta=10, \omega_{d}=0.2, \omega_{c}=0.1$, and $L=10$. (a) and $t=400 \frac{\ell^{2}}{D}$, respectively.

work. There the movement was due to treadmilling. One can include other aspects of filament assembly like nucleation or length-dependent depolymerization at the pointed end, which can induce further states [29]. However, the mean-field approach used here then becomes rather cumbersome and a full stochastic simulation might then be preferable.

The model is built on the assumption that all motor molecules are associated with plus ends. This is unlikely the case in cells. From earlier work, we know that the precise distribution of motors does not matter and even if the motor distribution is a dynamic field, the same states are generated
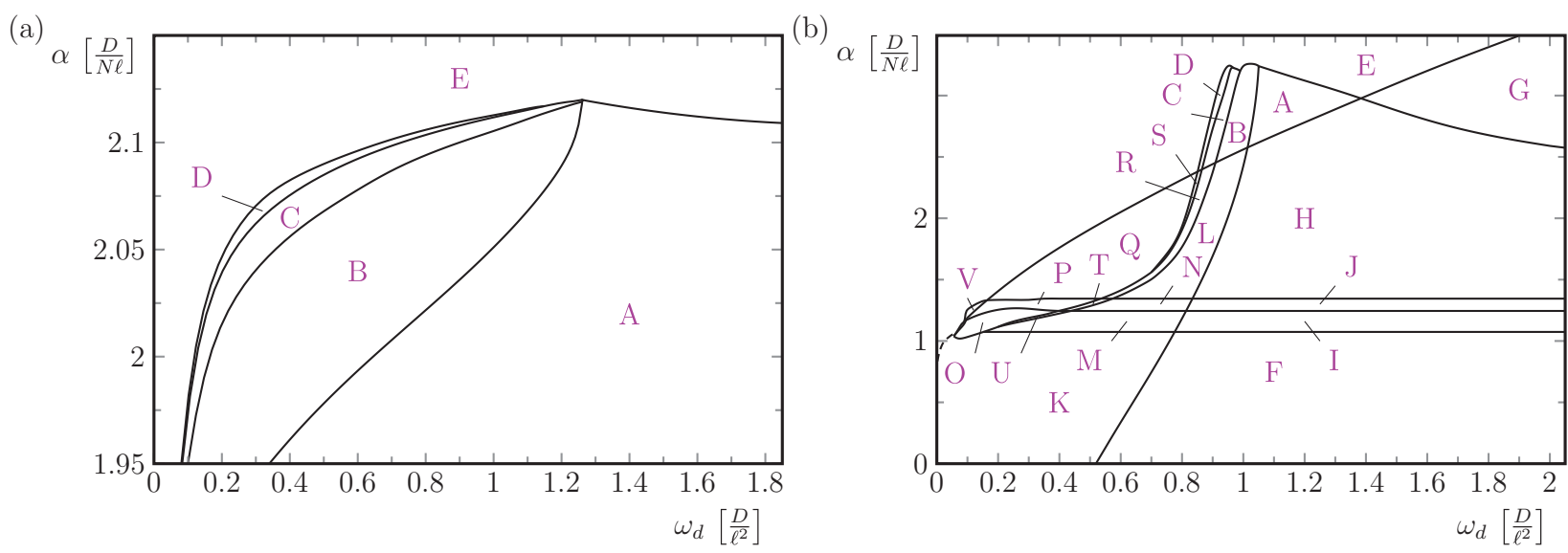

FIG. 13. Phase diagrams for $\beta=0.5$ (a) and for $\beta=10$ (b). The different phases are identified in Table I. There is no bifurcation between the phases $\mathrm{E}$ (cluster state $\mathrm{C}$ ) and $\mathrm{K}$ (stationary state $\mathrm{S}$ ). The dashed line marks the inflection point of the curve representing the states amplitude as a function of $\alpha$. Other parameter values are $\omega_{c}=0.1$ and $L=10$. 
TABLE I. Key to the phases shown in Fig. 13, indicating whether the states exist (s: stable; u: unstable) or not (-). The various states are homogenous (H), traveling clusters (TC, Fig. 12), clusters (C, Fig. 10), traveling waves (T, Fig. 11), stationary (S, Fig. 6), and oscillatory (O1, O2, Figs. 7 and 8).

\begin{tabular}{llllllll}
\hline \hline & $\mathrm{H}$ & $\mathrm{TC}$ & $\mathrm{C}$ & $\mathrm{T}$ & $\mathrm{S}$ & $\mathrm{O} 1$ & $\mathrm{O} 2$ \\
\hline $\mathrm{A}$ & $\mathrm{s}$ & - & $\mathrm{s}$ & - & - & - & - \\
$\mathrm{B}$ & $\mathrm{u}$ & - & $\mathrm{s}$ & - & $\mathrm{s}$ & - & - \\
$\mathrm{C}$ & $\mathrm{u}$ & - & $\mathrm{s}$ & - & - & $\mathrm{s}$ & - \\
$\mathrm{D}$ & $\mathrm{u}$ & - & $\mathrm{s}$ & - & - & - & $\mathrm{s}$ \\
$\mathrm{E}$ & $\mathrm{u}$ & - & $\mathrm{s}$ & - & - & - & - \\
$\mathrm{F}$ & $\mathrm{s}$ & - & - & - & - & - & - \\
$\mathrm{G}$ & $\mathrm{u}$ & $\mathrm{s}$ & - & - & - & - & - \\
$\mathrm{H}$ & $\mathrm{s}$ & $\mathrm{s}$ & - & - & - & - & - \\
$\mathrm{I}$ & $\mathrm{s}$ & - & - & $\mathrm{s}$ & - & - & - \\
$\mathrm{J}$ & $\mathrm{s}$ & $\mathrm{s}$ & - & $\mathrm{s}$ & - & - & - \\
$\mathrm{K}$ & $\mathrm{u}$ & - & - & - & $\mathrm{s}$ & - & - \\
$\mathrm{L}$ & $\mathrm{u}$ & $\mathrm{s}$ & - & - & $\mathrm{s}$ & - & - \\
$\mathrm{M}$ & $\mathrm{u}$ & - & - & $\mathrm{s}$ & $\mathrm{s}$ & - & - \\
$\mathrm{N}$ & $\mathrm{u}$ & $\mathrm{s}$ & - & $\mathrm{s}$ & $\mathrm{s}$ & - & - \\
$\mathrm{O}$ & $\mathrm{u}$ & - & - & $\mathrm{s}$ & - & - & - \\
$\mathrm{P}$ & $\mathrm{u}$ & $\mathrm{s}$ & - & $\mathrm{s}$ & - & - & - \\
$\mathrm{Q}$ & $\mathrm{u}$ & $\mathrm{s}$ & - & - & - & - & - \\
$\mathrm{R}$ & $\mathrm{u}$ & $\mathrm{s}$ & - & - & - & $\mathrm{s}$ & - \\
$\mathrm{S}$ & $\mathrm{u}$ & $\mathrm{s}$ & - & - & - & - & $\mathrm{s}$ \\
$\mathrm{T}$ & $\mathrm{u}$ & $\mathrm{s}$ & - & $\mathrm{s}$ & - & $\mathrm{s}$ & - \\
$\mathrm{U}$ & $\mathrm{u}$ & - & - & $\mathrm{s}$ & - & $\mathrm{s}$ & - \\
$\mathrm{V}$ & $\mathrm{u}$ & - & $\mathrm{s}$ & $\mathrm{s}$ & - & - & - \\
\hline \hline & & & & & & & \\
\hline
\end{tabular}

than if it is imposed [17]. Furthermore, the data on yeast cells presented in Ref. [18] and the observations made in Refs. [9,24] suggest that motors can accumulate at filament plus ends. Introducing a dynamic motor distribution would again make the description rather cumbersome such that a full stochastic simulation might be favoured to study its effects.

The dynamic accumulation of myosin at plus ends could also provide the molecular basis for bipolar filament formation. In Ref. [15], formin, an actin nucleating and polymerization promoting protein that is associated with actin-filament plus ends, was reported to co-localize with myosin clusters. It is also associated with myosin "nodes" that are observed in fission yeast [18]. Motor-mediated accumulation of formins would naturally explain the emergence of bipolar filaments. Note furthermore that these experimental data together with our theory strongly suggest that the distribution of myosin clusters reveals the polar order of filaments in contractile rings, which is difficult to assess otherwise. We would also like to point out that the values $\omega_{d}$ and $\omega_{c}$ of bipolar filament breakup and formation do not directly determine the fraction of bipolar filaments. This is only true in the homogenous stationary state. For example, for the state presented in Fig. 6, where $\omega_{c}=0.1, \omega_{d}=0.5$, and the average concentration of plus- and minus-filaments is roughly 0.5 , we expect for the average concentration of bipolar filaments $c_{0}^{\mathrm{bp}} \approx \omega_{d} c_{0}^{+} c_{0}^{-} / \omega_{c}=0.05$, which is close to the value obtained in the solution to the dynamic equations. In contrast, for the parameters used in Fig. 12, where now $\omega_{d}=0.2$, we find $c_{0}^{\mathrm{bp}} \approx \omega_{d} c_{0}^{+} c_{0}^{-} / \omega_{c} \approx 0.13$, whereas the actual average concentration of bipolar filaments is very close to zero.
Let us finish by relating the states that are generated by our dynamic equations to the experimental observations made on fission yeast that are presented in Fig. 2. Salient aspects of cluster dynamics highlighted in Fig. 2 are also present in the various states we reported above. Clockwise and counter clockwise motion is present in the traveling state T, Fig. 11. Symmetric fusion is associated with several states, namely the cluster state C, Fig. 10, and traveling cluster state TC, Fig. 12, whereas the oscillatory states $\mathrm{O} 1$ and $\mathrm{O} 2$ show elongation and separation of clusters, Fig. 7. Asymmetric fusion is not readily detected in the asymptotic states of our dynamic system but was observed in Ref. [15], when treadmilling was accounted for. These comparisons further support the idea that the distribution of actin polarity within actomyosin rings could be inferred from the dynamics of myosin clusters, in vivo and in vitro.

\section{ACKNOWLEDGMENTS}

We thank V. Wollrab, A. Wald, and R. Thiagarajan for lively discussions, and we gratefully acknowledge supported by the German Research Foundation (DFG) through the Collaborative Research Center SFB 1027.

\section{APPENDIX: DYNAMICS EQUATIONS FROM WOLLRAB et al. [Nat. Commun. 7, 11860 (2016)]}

Here, we compare in detail the dynamic Eqs. (1)-(11) to the ones introduced in Ref. [15]. The latter are

$$
\partial_{t} c^{+}=D \partial_{x}^{2} c^{+}-v \partial_{x} c^{+}-\partial_{x} J^{+}-\omega_{c} c^{+} c^{-}+\omega_{d} c^{\mathrm{bp}},
$$

$$
\begin{gathered}
\partial_{t} c^{-}=D \partial_{x}^{2} c^{-}+v \partial_{x} c^{-}-\partial_{x} J^{-}-\omega_{c} c^{+} c^{-}+\omega_{d} c^{\mathrm{bp}}, \\
\partial_{t} c^{\mathrm{bp}}=D \partial_{x}^{2} c^{\mathrm{bp}}-\partial_{x} J^{\mathrm{bp}}+\omega_{c} c^{+} c^{-}-\omega_{d} c^{\mathrm{bp}}
\end{gathered}
$$

with

$$
\begin{aligned}
J^{ \pm \pm}=\alpha \int_{0}^{\ell} c^{ \pm}(x)\left[c^{ \pm}(x+\xi)-c^{ \pm}(x-\xi)\right] d \xi \\
J^{ \pm \mp}=\mp \beta \int_{0}^{\ell} c^{ \pm}(x) c^{\mp}(x \mp \xi) d \xi \\
J^{ \pm \mathrm{bp}}= \pm \alpha \int_{0}^{\ell} c^{ \pm}(x) c^{\mathrm{bp}}(x \pm \xi) d \xi \\
\mp \beta \int_{0}^{\ell} c^{ \pm}(x) c^{\mathrm{bp}}(x \mp \xi) d \xi \\
J^{\mathrm{bp} \pm}=\mp \alpha \int_{0}^{\ell} c^{\mathrm{bp}}(x) c^{ \pm}(x \mp \xi) d \xi \\
\pm \beta \int_{0}^{\ell} c^{\mathrm{bp}}(x) c^{ \pm}(x \pm \xi) d \xi
\end{aligned}
$$

$$
J^{\mathrm{bpbp}}=\alpha \int_{0}^{\ell} c^{\mathrm{bp}}(x)\left[c^{\mathrm{bp}}(x+\xi)-c^{\mathrm{bp}}(x-\xi)\right] d \xi
$$


Equations (A1) and (A2) contain currents $\pm v c^{ \pm}$that account for treadmilling. Although this term can shift phase boundaries, we did not find it to generate qualitatively new states. The interaction strength between bipolar filaments in $J^{\text {bpbp }}$ differs by $\beta / 2$ from the one in Eq. (11). We have added the dependence on $\beta$, because also for bipolar filaments there is an interaction through motors linking filaments of opposite orientation. Similarly, in Eqs. (9) and (10) we have added terms proportional to $\alpha$ in the currents $J^{ \pm \mathrm{bp}}$ and $J^{\mathrm{bp} \pm}$ compared to Eqs. (A5) and (A6) to capture the contribution of interactions between parallel filaments in case there is an antiparallel overlap. As before, we did not find these changes to introduce qualitatively new states. The factor $1 / 2$ present in the current $J^{\mathrm{bp} \pm}$, Eq. (10), but absent in Eq. (A6) accounts for the reduced mobility of bipolar filaments compared to polar filaments.

The two sets of dynamic equations become essentially equal in the case $v=0, \beta \gg \alpha$, and $c_{0}^{ \pm} \gg c_{0}^{\mathrm{bp}}$. This condition holds approximately for the phase diagram in Fig. 13(b), where $\beta>$ $3 \alpha$ and $\omega_{d}>\omega_{c}$ for most of the diagram.
[1] B. Alberts, A. Johnson, J. Lewis, M. Raff, K. Roberts, and P. Walter, Molecular Biology of The Cell, 5th ed. (Garland Science, Oxford, UK, 2008)

[2] D. Bray, Cell Movements, From Molecules to Motility, 2nd ed. (Taylor \& Francis, Oxford, UK, 2001)

[3] C. Schwayer, M. Sikora, J. Slováková, R. Kardos, and C.-P. Heisenberg, Dev. Cell 37, 493 (2016).

[4] M. Fritzsche, A. Lewalle, T. Duke, K. Kruse, and G. Charras, Mol. Biol. Cell 24, 757 (2013).

[5] K. Kruse and F. Jülicher, Phys. Rev. Lett. 85, 1778 (2000).

[6] M. Lenz, T. Thoresen, M. L. Gardel, and A. R. Dinner, Phys. Rev. Lett. 108, 238107 (2012).

[7] S. Wang and P. G. Wolynes, Proc. Natl. Acad. Sci. U.S.A. 109, 6446 (2012).

[8] M. Lenz, Phys. Rev. X 4, 041002 (2014).

[9] J. M. Belmonte, M. Leptin, and F. Nédélec, Mol. Syst. Biol. 13, 941 (2017).

[10] T. Thoresen, M. Lenz, and M. L. Gardel, Biophys. J. 100, 2698 (2011).

[11] H. Ennomani, G. Letort, C. Guérin, J.-L. Martiel, W. Cao, F. Nédélec, E. M. De la Cruz, M. Thery, and L. Blanchoin, Curr. Biol. 26, 616 (2016).

[12] D. Riveline and A. Buguin, Devices and Methods for Observing the Cell Division, Patent No. WO/2010/092116 (2010-08-19).

[13] V. Wollrab and D. Riveline, Devices and Methods for Observing Eukaryotic Cells without Cell Wall, Patent No. WO/2013/135809 (2015-02-12).

[14] D. Riveline, Methods for Observing Cells with Cell Wall or Invertebrate Embryos with Oblong Eggshell, Patent No. WO/2013/144302 (2015-03-19).
[15] V. Wollrab, R. Thiagarajan, A. Wald, K. Kruse, and D. Riveline, Nat. Commun. 7, 11860 (2016).

[16] K. Kruse, S. Camalet, and F. Jülicher, Phys. Rev. Lett. 87, 138101 (2001).

[17] K. Kruse and F. Jülicher, Phys. Rev. E 67, 051913 (2003).

[18] D. Vavylonis, J.-Q. Wu, S. Hao, B. O'Shaughnessy, and T. D. Pollard, Science 319, 97 (2008).

[19] M. Rauzi, P.-F. Lenne, and T. Lecuit, Nature 468, 1110 (2010).

[20] W. Luo, C.-H. Yu, Z. Z. Lieu, J. Allard, A. Mogilner, M. P. Sheetz, and A. D. Bershadsky, J. Cell Biol. 202, 1057 (2013).

[21] D. V. Köster, K. Husain, E. Iljazi, A. Bhat, P. Bieling, R. D. Mullins, M. Rao, and S. Mayor, Proc. Natl. Acad. Sci. U.S.A. 113, E1645 (2016).

[22] M. Nishikawa, S. R. Naganathan, F. Jülicher, and S. W. Grill, eLife 6, e19595 (2017).

[23] D. Goswami, K. Gowrishankar, S. Bilgrami, S. Ghosh, R. Raghupathy, R. Chadda, R. Vishwakarma, M. Rao, and S. Mayor, Cell 135, 1085 (2008).

[24] A. B. Verkhovsky, T. M. Svitkina, and G. G. Borisy, J. Cell Sci. 110, 1693 (1997).

[25] D. Riveline, A. Ott, F. Jülicher, D. A. Winkelmann, O. Cardoso, J. J. Lacapere, S. Magnusdottir, J. L. Viovy, L. Gorre-Talini, and J. Prost, Eur. Biophys. J. 27, 403 (1998).

[26] J. Prost, J. F. Joanny, and J. M. R. Parrondo, Phys. Rev. Lett. 103, 090601 (2009).

[27] T. B. Liverpool and M. C. Marchetti, Europhys. Lett. 69, 846 (2005).

[28] C. Erlenkämper and K. Kruse, J. Chem. Phys. 139, 164907 (2013).

[29] P. Guthardt Torres, K. Doubrovinski, and K. Kruse, Europhys. Lett. 91, 68003 (2010). 